\title{
Clinical Trials of Classical Homeopathy: Reflections on Appropriate Research Designs
}

\author{
MENACHEM OBERBAUM, M.D., M.F.Hom., ${ }^{1}$ GEORGE VITHOULKAS, ${ }^{2}$ \\ ROBBERT VAN HASELEN, M.Sc. ${ }^{3}$
}

\begin{abstract}
The growing popularity of complementary medicine has been accompanied by a call for controlled clinical studies to examine the efficacy and validity of its various methods. The difficulties encountered in applying the evaluation methods of conventional medicine to complementary medicine are the result of the different paradigms underlying these two methods of medicine, and the differences in the healing process. This paper attempts to bridge the gap between these two approaches and to suggest a possibility to use conventional research methodology in clinical studies of classical homeopathy. Two methods are described. One is the randomization into a placebo or a treatment group after an individual remedy is chosen for each patient. This method requires an experienced homeopath and is reproducible only by the same homeopath in the same population. On the other hand, the expected success rate will be high. Another method is prescribing and treating according to so-called keynotes, a set of symptoms known to respond to a particular remedy that must be present in a patient in order to elicit a reaction from the remedy. This method is more suitable to a conventional design of a clinical study and is reproducible by anyone. Yet the expected success rate is much lower. Some general design issues that may have a particular impact on clinical trials of classical homeopathy are discussed, including the need for sufficiently powered trials to detect relatively small effects, strategies to deal with patient preference and embracing the use of "active-control" pragmatic trial designs.
\end{abstract}

\section{INTRODUCTION}

D uring the past few years, complementary and alternative medicine (CAM) has seen an increase in use (Eisenberg et al., 1993, 1998; Fisher and Ward, 1994) and interest (Astin et al., 1998; Fontanarosa and Lundberg, 1998; Hopper and Cohen, 1998). With this revival, there is also a growing demand for well-performed clinical studies that examine the effi- cacy and validity of the disciplines of complementary medicine (Glass, 1997; Fontanarosa and Lundberg, 1998).

Most forms of CAM differ from conventional medicine in their basic paradigm. A good example is homeopathy. Conventional medicine sees a disease as a sum of symptoms common to all pathologic conditions carrying the same name of the disease. In contrast, classical homeopathy sees a disease as a pathologic condition

\footnotetext{
${ }^{1}$ The Institute of Research on Complementary Medicine and The Center of Integrated Complementary Medicine, Shaare Zedek Medical Center, Jerusalem, Israel.

${ }^{2}$ The International School of Classical Homeopathy, Alonisos, Greece.

${ }^{3}$ The Royal London Homoeopathic Hospital, Great Ormond Street, London, UK.
} 
specific to a certain person (Hahnemann, 1994; Kent, 1991), an internal disease (Hahnemann, 1994) manifested by the sum of symptoms (Hahnemann, 1994) mental, emotional, and physical (Taylor et al., 2000) exhibited by the patient. As such, every sick person suffers from a unique disease, and the goal is to prescribe individually tailored remedies in order to stimulate self-healing or cure at the broadest possible level. In conventional medicine it is therefore possible to examine the influence of a $\beta$-blocker on a group of hypertensive patients, but it is not possible to do the same with a homeopathic remedy. Furthermore, the dynamics of the curative process differ. Whereas a hypertensive patient might (or might not) react with a decrease in blood pressure after receiving a conventional antihypertensive drug, the end point of the homeopathic remedy will be reached in a more complicated way. The patient's symptoms might be aggravated initially (Vithoulkas, 1980), the symptoms may disappear, old symptoms may reappear (Vithoulkas, 1980), or the patient may need to change remedies (Hahnemann, 1994; Kent, 1993b; Vithoulkas, 1980) until a cure is achieved.

A commentary in the Archives of Internal Medicine (Ernst and Kaptchuk, 1996) called for more research in homeopathy, putting aside for the moment its implausibility. Accepting the need for more clinical trials of homeopathy, we would like to make the case that some modifications of the conventional requirements for clinical studies are required, particularly in studies using classical homeopathy (and indeed other types of CAM) where treatment is highly individualized. For instance, the evaluation of the outcome should take into account factors such as initial aggravation of the disease, the appearance and disappearance of old symptoms, and the need to change remedies.

In order to avoid confusion it should be noted that there are three possible types of clinical trials of homeopathy:

1. Clinical trials of complexes (mixture of homeopathic dilutions, usually low dilutions, targeting one specific disease).

2. Clinical trials of isotherapy (dilutions of the pathologic agent, targeting one specific disease).
3. Clinical trials of classical homeopathy, which is considered the genuine unadapted form of homeopathy.

Whereas the rules for clinical trials with complexes and isotherapy could be the same as for conventional trials, this paper principally refers to classical homeopathy and indirectly to other forms of CAM where treatment is highly individualized.

Ignoring the major basic differences between homeopathy and conventional medicine can lead to failure in planning clinical trials (Senn, 1997) and misinterpretation of the results. For example, initial symptom aggravation can be misinterpreted as failure. Such initial aggravations have been observed in clinical homeopathy for more than 200 years, and a recent placebo-controlled trial indicated that initial aggravations are likely to be more than a nocebo response and are associated with a subsequent favorable outcome (Shipley, 1983).

This paper attempts to raise some important points and suggests certain requirements for clinical studies in homeopathy, especially classical homeopathy. Keeping them in mind while planning such clinical studies will result in fewer pitfalls, and facilitate the necessary valid evaluation of the efficacy of classical homeopathy and other highly individualized types of CAM.

\section{IMPLICATIONS OF MAIN PRINCIPLES OF CLASSICAL HOMEOPATHY FOR THE DESIGN OF CLINICAL TRIALS}

One of the main principles of homeopathy, if not the most important one, is the need for individualization (Hahnemann, 1994; Vithoulkas, 1980). The patient has to be characterized by qualities and symptoms that are unique, and that differ from those of other patients suffering from the same clinical disease. Contrary to conventional medicine, "idiosyncrasy" is a crucial element in this form of therapy. Modified for homeopathy, "idiosyncrasy" is defined as the complex of mental, emotional, and physical pathologic peculiarities of the patient. There is no possibility of using a specific remedy for a specific indication. For all the 
above reasons there are two possible methods of structuring a homeopathic clinical study. These approaches are evaluated in hierarchical order.

\section{Unrestricted prescribing}

The best method, if we do not want to restrict the possibilities, would be for an experienced practitioner to assess every case in the study and determine which remedy is indicated for each case. After the determination of the indicated remedies, the patients would be randomly assigned to either a placebo or treatment group. This method, however, requires a skilled homeopath in order to demonstrate the full therapeutic possibilities of classical homeopathy. In addition to being logistically complex, this kind of study is truly reproducible only by the same homeopath within the same population. A change of the treating homeopath will change the results, for the better or worse, depending on his or her skills. Therefore, from the point of view of reproducibility, this technique is not optimally suited for a classical conventional design for clinical trials in homeopathy. On the other hand, this kind of study will yield the best results.

Aside from the expertise of the homeopath, the indication and the severity of the pathology will also determine the success rate. Based on our experience, the pathologic conditions for which the best results would be expected include (from 1 to 7 in descending order):

1. Frontal and (less so) maxillary sinusitis.

2. Early stages of chronic and acute liver diseases.

3. Infertility (female factor).

4. Epilepsy (grand mal) in children and (less successfully) in adults.

5. Acute arthritis (monoarthritis).

6. Irritable bowel syndrome.

7. Chronic prostatitis.

\section{Keynote prescribing}

A more suitable design for classical medical research, but one that is expected to achieve poorer results, would be to agree on prescribing remedies only according to keynotes. This is based on the knowledge and experience that a certain cluster of symptoms that patients exhibit respond to a particular remedy. Only preselected patients showing these "group keynote symptoms" would participate in such a study. The keynotes have to be selected by an experienced homeopath or panel of homeopaths, and modified for different nosological entities.

An example of such keynote prescribing is a clinical trial of the homeopathic treatment of premenstrual syndrome (PMS) with the remedy Lachesis in patients who exhibit the following cluster of symptoms (Yakir, 2001):

1. Amelioration of the PMS as soon as the blood flow starts.

2. Sensitivity to heat.

3. Aggravation of the symptoms on falling asleep.

4. Sensitivity to the touch of the throat and/or intolerance of the pressure of a tight collar.

All patients with these symptoms would be randomly assigned to receive either Lachesis or placebo.

Because the practice of using keynotes in a clinical experiment is not common, and there are no generally accepted keynotes for particular indications in the homeopathic literature, the keynote selection process would be pioneering, and would have to be based on extensive experience. Only an experienced homeopath or a panel of experienced homeopaths would be suitable for the selections of the keynote symptoms.

\section{APPROPRIATE METHODS: FURTHER CONSIDERATIONS}

To evaluate the effect of a homeopathic remedy correctly certain rules must be acknowledged and adhered to throughout the clinical trial. Such rules concern the different possible reactions in different patients receiving the homeopathic remedy: a chronic disease will need a longer treatment period until the remedy's effect has waned, and therefore, a longer observation time than for a disease that has been present for a brief time.

An initial aggravation period is likely to oc- 
cur (Hahnemann, 1994; Vithoulkas,1980). If it does appear, it will be expected within the first month after the application of the homeopathic remedy. Because initial aggravations are more likely to occur after active homeopathic remedies than after placebo (nocebo responses), such occurrences can be used as a secondary outcome measure in trials. The severity and length of the aggravation is directly related to the duration of the disease and the health condition of the patient (Hahnemann, 1994). A patient in a depleted condition will suffer from a stronger and more prolonged aggravation. Yet this aggravation is an integral, necessary, and usually desirable stage in the curative process (Vithoulkas, 1980). Because it is a response associated with a favorable outcome, it should not be stopped or interfered with by conventional drug treatment (except for simple analgesics such as acetylsalicylic acid and paracetamol), unless the reaction endangers the life of the patient.

For clinical trials of classical homeopathy, it is preferable to study patients who have not taken many conventional medications in the past, and who are not taking long-term medications at the present time. The use of antibiotics during the month preceding the trial, or the use of steroids during the 3-month period prior to the trial, or a history of long-term steroid use in the past, especially in the 6-9 months preceding the clinical trial, should be cause for exclusion from the study. Antibiotics and steroids are considered as the most disruptive and disturbing drugs to the process of homoeopathic healing (Vithoulkas 1980).

It is preferable that during the clinical trial, conventional medications be used only in emergency cases. The only medications allowed are acetylsalicylic acid and paracetamol. On the other hand, it is also desirable for participants in a classical homeopathic clinical trial not to have received many homeopathic remedies in the past. The unsuccessful administration of many homeopathic remedies can complicate the pathologic picture by adding new symptoms, thereby masking the real symptoms of the disease.

In light of the abovementioned considerations, the pathologic conditions most suitable for clinical research in classical homeopathy, include:

1. Diseases for which there is no conventional treatment, or diseases for which conventional treatment is insufficient (e.g., acute liver diseases, early stages of chronic liver diseases, irritable bowel syndrome, pancreatitis, Ménière's disease, aphthous stomatitis).

2. Diseases that are not life-threatening (e.g., infertility, childhood behavioral problems, psoriasis).

More severe and life-threatening diseases can be included in a clinical trial program at a later time, but initially we suggest proceeding with diseases that fulfill the abovementioned conditions.

We suggest that observation not be stopped even if it first appears that there is no clinical improvement. Because the curative process is a dynamic one that moves through many stages, any change of symptoms justifies the continuation of the treatment (e.g., during the treatment of a female's headache, leukorrhea can appear; in the treatment of asthma, atopic dermatitis may appear before the asthma resolves).

According to the homeopathic disease model, a person who is ill should be regarded as covered with pathologic "layers" (Vithoulkas, 1980). This means that a long-lasting disease will be equal to a "many layered disease" (Vithoulkas, 1980), and the cure for a long-lasting disease might, in many cases, require more than one remedy, one remedy per layer, and a prolonged treatment period.

Concerning the repetition of remedies, certain rules should be adhered to. Contrary to conventional logic, a homeopathic remedy that is more dilute, and therefore contains less substance (in homeopathic terminology, "higher potency"), is longer acting, and has stronger mental and emotional effects than a lower potency (less diluted homeopathic remedy) (Kent, 1993a). Therefore, as a rule, low potencies can be repeated often; with increasing potency the number of repetitions per unit time should be decreased (Vithoulkas, 1980). 
Because the repertoire of the homeopathic medicines includes thousands of remedies, and the decision as to which remedy should be used might also include elements of uncertainty, there is the possibility of choosing the wrong remedy. Consequently, it may be necessary to change the remedy more than once until the correct remedy is found. In any clinical study using unrestricted prescribing, changing the remedy should be allowable. Maintaining double-blinded conditions while allowing unrestricted prescribing, can be accomplished by preparing for each patient a kit that contains either the most commonly used remedies or placebo remedies according to a randomization number list. Alternatively, a person blinded to both the patient's condition and to the remedy prescribed by the homeopath can administer the tablet to each patient, which according to a randomization number list will be either active remedy or placebo.

According to the homeopathic understanding of health and disease, in many cases the mind and the emotions, as well as the body are affected (Vithoulkas, 1980). Therefore, when evaluating the success or failure of the treatment, the well-being of the patient has to be considered, in addition to the evaluation of the changes of the physical symptoms (Vithoulkas, 1980). Cases with mental and emotional improvement should be evaluated as partial successes because often an improvement in the general emotional and mental condition occurs and often precedes an improvement in the physical complaints.

\section{SOME GENERAL METHODOLOGICAL REFLECTIONS}

For many years the prevailing opinion was that clinical trials of classical homeopathy were impossible because of the highly individualized nature of prescribing. It has now become clear that this is not the case. Although homeopathy cannot claim to be a "special case," there are some general design issues that may have particular impact on clinical trials of classical homeopathy (van Haselen, 1998).
The homeopathic interview is in itself an indepth psychologic review of the patient as a whole. A patient's assessment of his or her own utility (need fulfillment) is likely to be a result of the process of the consultation itself as well as of the actual remedy given. It is quite conceivable that this minimizes the difference between the active and the placebo homeopathic treatment and that the "true" effect is relatively small. Although this problem is not unique to classical homeopathy, trials will require sufficient power to demonstrate such small effects.

It is now emerging (McKee, 1999) that nonrandomized studies do not provide systematically different measurements of treatment effects compared to randomized controlled trials (RCTs). Effects may be greater in RCTs if patients enrolled received higher quality of care compared to patients in nonrandomized studies. However, RCTs may produce a lower estimate of treatment effect for several reasons (McKee, 1999). One reason for this, which may be of particular relevance for trials of homeopathy, is the aspect of patient preference. Trials are considered to be indicated and ethical when there is collective professional equipoise (uncertainty) about the efficacy of a particular therapy. If patients share this equipoise, most would be willing to be randomized. However in the real world, patients will have a clear preference (whether evidence-based or not) and this is certainly applicable to patients seeking treatment by a classical homeopath. As a consequence, patients with a clear preference will not enter clinical trials, which then comprise a selection of patients with "individual equipoise." Little is known about the complex interaction between patient preference and treatment outcome, and even trials with separate preference arms may not be appropriate tools to disentangle these effects (Senn, 1997). The potential impact of preference on the results of clinical trials is even greater where the true effects are small and, as mentioned above, this is likely to be the case in trials of classical homeopathy.

Where does this leave us? A possible conclusion is that the pure effects of correctly individualized remedies are, to a certain extent, unknowable. Negative results of trials may 
well be false-negatives (Type I errors) as a result of a combination of the abovementioned mechanisms.

Are there possible solutions to this dilemma? A partial solution could be to record the preference of a patient prior to randomization. This will enable an analysis of the influence of preference on outcome (Moffett et al., 1999), and adjustment for this if necessary. This would be a useful contribution to the current debate on the role of preference.

Another possible solution is to move away from randomization and frequentist statistics to the use of Bayesian methods (Senn, 1997; Vallance, 1998) in large observational data sets where outcome and most relevant prognostic variables have been recorded (Senn, 1997). However, most researchers wish to maintain the ideal of randomization, either with or without blinding. In the case of placebo-controlled trials of classical homoeopathy, patient willingness to be randomized could be improved by offering all patients active homeopathic treatment after completion of the trial phase. This will provide all patients with the assurance that they will receive active homeopathic treatment, either immediately or after completion of the trial phase. In other cases, it may be necessary to shift the emphasis from placebocontrolled explanatory trials to more pragmatic trials (Schwartz and Lellouch, 1967) where the added value of homeopathic management is investigated. For instance, patients with early stages of common chronic diseases such as asthma or rheumatic diseases could be randomly assigned to receive either standard conventional management by their general practitioner or standard conventional management plus treatment with classical homeopathy. The observational period in such a study should be long enough (minimally 1 year) to enable the evaluation of the cost effectiveness of classical homeopathy. Such a trial would evaluate the process of treatment with classical homeopathy rather than the pure effects of individually prescribed remedies.

Such trials would not only be focused on disease severity as an outcome measure, but also on changes in health-related quality of life, and when relevant, changes in the use of conventional medicines. Such trials will by necessity yield much more heterogeneous data (representative of the "messiness" of the real world), and could be hampered by study subjects being lost to follow-up. Therefore, it is clear that such trials have limitations and problems of their own. Because of the specific nature of the homeopathic consultation, the process of care may be an important factor contributing to the overall utility (need fulfillment) gained by patients (Buxton, 2000). The characteristics of treatment that patients value and the relative values of these characteristics could be further investigated with the technique of conjoint analysis. (Ryan and Shelley, 2000). Such an approach is contrary to explanatory trials of classical homeopathy, where process utility is regarded as mere inconvenient placebo interference.

\section{CONCLUSIONS}

Homeopathy is a non-Cartesian art of medicine and as such there are difficulties in its being evaluated using scientific methods, developed and used by Newtonian-Cartesian scientific modern medicine. Nevertheless it is possible to establish a link between both systems of medicine, provided there is a willingness by both to respect the differences and the needs of the other. In particular, conventional medicine will have to accept the need for individualization, and acknowledge that a disease can be seen as a unique set of symptoms expressed by individual patients. In homeopathy, on the other hand, it is important to acknowledge the need for reproducible evidence from clinical trials, which is one of the cornerstones of conventional evidence-based medicine. These principles are, of course, also applicable for other CAM modalities in which highly individualized treatment is central.

In conclusion, it is important to address the pure efficacy of homeopathic medicines in placebo-controlled trials. However, a one-sided emphasis on obtaining only efficacy data is not justified. Other types of evidence such as cost effectiveness and safety data should be obtained as well (van Haselen, 1998) if homeopathy is to merit the status within integrated health care it deserves. 


\section{ACKNOWLEDGMENT}

The authors are grateful to Dr. Shepard Schwartz from the Department of Pediatrics, Shaare Zedek Medical Center, Jerusalem, for his help in editing the final version of this paper, and his useful comments.

\section{REFERENCES}

Astin JA, Marie A, Pelletier KR, Hansen E, Haskell WL. A review of the incorporation of complementary and alternative medicine by mainstream physicians. Arch Intern Med 1998;158:2303-2310.

Buxton M. Assessing the cost-effectiveness of homeopathic medicines: Are the problems different from other health technologies? Br Homoeopath J 2000;89 (suppl): S20-S22.

Eisenberg DM, Davis RB, Ettner SL, Appel S, Wilkey S, Van Rompay M Kessler RC. Trends in alternative medicine use in the United States 1990-1997: Results of a follow-up national survey. IAMA 1998;280:1569-1575.

Eisenberg DM, Kessler RC, Foster C, Norlock FE, Calkins DR, Delbanco TL. Unconventional medicine in the United States. Prevalence, costs, and patterns of use. N Engl J Med 1993;328:246-252.

Ernst E, Kaptchuk TJ. Homeopathy revisited. Arch Intern Med 1996;28;156:2162-2164.

Fisher P, Ward A. Complementary medicine in Europe. BMJ 1994;309:107-111.

Fontanarosa PB, Lundberg GD. Alternative medicine meets science. JAMA 1998;280:1618-1619.

Glass TA, Kushi LH, Schuck JR, Steele L, Jonas WB. Quantitative methods in research on complementary and alternative medicine: A methodological manifesto. Med Care 1997;35:1079-1094.

Hahnemann S. Organon of Medicine, 6th ed. New Delhi: B. Jain Publishers Ltd., 1994.

Hopper I, Cohen M. Complementary therapies and the medical profession: A study of medical student's attitudes. Altern Ther Health Med 1998;4:68-73.

Kent JT. Lectures on Homeopathic Materia Medica. New Delhi: B. Jain Publishers, 1991:177, 196-198.

Kent JT. Lectures on Homoeopathic Philosophy. B. Jain Publishers, New Delhi, 1993a:92-93.

Kent JT. Lectures on Homoeopathic Philosophy. New Delhi: B. Jain Publishers, 1993b:239-240.
McKee M, Britton A, Black N, McPherson K, Sanderson $\mathrm{C}$, Bain C. Interpreting the evidence: Choosing between randomised and non-randomised studies. BMJ 1999; 319:312-315.

Moffett JK, Torgerson D, Bell-Syer S, Jackson D, LlewlynPhillips H, Farrin A, Barber J. Randomised controlled trial of exercise for low back pain: Clinical outcomes, costs, and preferences. BMJ 1999;319:279-283.

Ryan M, Shelley F. Using conjoint analysis to elicit preferences for health care. BMJ 2000;320:1530-1533.

Schwartz D. Lellouch J. Explanatory and pragmatic attitudes in therapeutical trials. I Chron Dis 1967;20: 637-648.

Senn S. Statistical issues in drug development. Chichester: John Wiley \& Sons, 1997:43-54.

Shipley M, Berry H, Broster G. Controlled trial of homoeopathic treatment of osteoarthritis. Lancet 1983;1: 97-98.

Taylor MA, Reilly D, Llewellyn-Jones RH, McSharry C, Aitchison TC. Randomised controlled trial of homeopathy versus placebo in perennial allergic rhinitis with overview of four trial series. BMJ 2000;321:471-476.

Vallance AK. Can biological activity be maintained at ultra-high dilution? An overview of homeopathy, evidence, and Bayesian philosophy. J Altern Complement Med 1998;4:49-76.

van Haselen RA. Randomised, placebo controlled trials of classical homoeopathy: Is there a problem? $\underline{\mathrm{Br} H o-}$ moeopath J 1998;97:128-130.

van Haselen RA, Fisher P. Evidence influencing British Health Authorities' decisions in purchasing complementary medicine. JAMA 1998;280:1564-1565.

Vithoulkas G, The Science of Homeopathy. New York: Grove Press Publishers, 1980.

Yakir M, Kreitler S, Brzezinski A, Vithoulkas G, Oberbaum M, Bentwich Z. Effects of homeopathic treatment in women with premenstrual syndrome: A pilot study. Br Homoeopath J 2001;90:148-153.

Address reprint requests to: Menachem Oberbaum, M.D., M.F.Hom. The Center for Integrated Complementary Medicine Shaare Zadek Medical Center P.O. Box 3235 Jerusalem 91031 Israel

E-mail: oberbaum@netvision.net.il 
This article has been cited by:

1. L. Hektoen, S. Larsen, S. A. Odegaard, T. Loken. 2005. Comparison of Homeopathy, Placebo and Antibiotic Treatment of Clinical Mastitis in Dairy Cows - Methodological Issues and Results from a Randomized-clinical Trial. Journal of Veterinary Medicine Series A 51:9-10, 439-446. [CrossRef]

2. Menachem Oberbaum, George Vithoulkas, Robbert van Haselen, Shepherd Singer . 2003. Reinventing the Wheel? Or the Emperor's New Clothes. The Journal of Alternative and Complementary Medicine 9:5, 613-615. [Citation] [PDF] [PDF Plus]

3. Vic Stambolović . 2003. Blind Trial: A Subjugation Tool. The Journal of Alternative and Complementary Medicine 9:5, 615-617. [Citation] [PDF] [PDF Plus]

4. Harald Walach . 2003. Reinventing the Wheel Will Not Make It Rounder: Controlled Trials of Homeopathy Reconsidered. The Journal of Alternative and Complementary Medicine 9:1, 7-13. [Citation] [PDF] [PDF Plus] 